\title{
Cardiac platypnea-orthodeoxia syndrome in a 73-year-old woman
}

\author{
Khai-Jing Ng MD, Yi-Da Li MD
}

A 73-year-old woman was admitted because of progressive shortness of breath over the previous two weeks, which affected her daily activities. She had a history of congestive heart failure (New York Heart Association functional class II), hypertension and scoliosis. Her medications included acetylsalicylic acid, amiloride, hydrochlorothiazide, atenolol, rosuvastatin and spironolactone. She had no orthopnea or productive cough. On physical examination, her heart sounds were normal, without murmur. An electrocardiogram showed normal sinus rhythm.

During the patient's stay in hospital, intermittent hypoxemia was noted when she was eating or sitting upright; hypoxemia improved when the patient was lying down. Oxygen saturation was found to step-down gradually, from $90 \%$ (when the patient was supine) to $78 \%$ ( $45^{\circ}$ recline) to $69 \%$ ( $90^{\circ}$ upright), before recovering to $87 \%$ (return to supine).

A transthoracic echocardiogram showed a mildly dilated left atrium with a mildly dilated aortic root $(3.95 \mathrm{~cm})$. No valvular abnormalities, pericardial effusion or atrial septal aneurysm were seen. Bubbles were seen in the left atrium and ventricle within three cardiac cycles on bubble echocardiography (Figure 1 and Appendix 1, available at www.cmaj.ca/lookup/suppl/ doi:10.1503/cmaj.141525/-/DC1), suggesting intracardiac shunting. Computed tomography of the chest showed no evidence of lung parenchymal disease or pulmonary embolism; however, scoliosis and a tortuous, dilated aortic root were noted. Right heart catheterization confirmed normal pulmonary pressure (mean pulmonary arterial pressure $12 \mathrm{~mm} \mathrm{Hg}$; mean right atrial pressure $4 \mathrm{~mm} \mathrm{Hg}$ ) and a normal Qp/Qs ratio (0.46). Direct measurement of oxygen saturation during cardiac catheterization showed a step-down pattern: peripheral arterial saturation $\left(\mathrm{SPO}_{2}\right)$ in pulmonary capillary wedge position, left ventricle and aorta were $99.7 \%, 85.5 \%$ and $88.6 \%$, respectively, which is compatible with the presence of intracardiac right-to-left shunt through a patent foramen ovale. We diagnosed platypneaorthodeoxia syndrome.

The patient was scheduled for transfer to a tertiary care centre for further surgical intervention. Because her oxygen saturation fluctuated between $80 \%-90 \%$ in the supine position, we questioned whether she would be able to tolerate the three-hour journey. Considering the pathophysiology of platypnea-orthodeoxia syndrome, we attempted to increase left ventricular enddiastolic pressure using a vasopressor to improve right-to-left shunting. We initially used low-dose dopamine $(5 \mu \mathrm{g} / \mathrm{kg}$ per min). Unfortunately, tachycardia developed, and the patient's dyspnea and intermittent desaturation did not improve. The patient also felt chest discomfort from palpitations. We changed the vasopressor to norepinephrine $(5 \mu \mathrm{g} / \mathrm{hr})$, which improved the patient's condition - her oxygen saturation was greater than $95 \%$ when she was in a supine position.

The patient was successfully transferred to the tertiary care hospital, where she underwent implantation of a septal occluder. A follow-up cardiac echocardiogram showed no residual shunting, and the patient's dyspnea decreased substantially after closure of the patent foramen ovale (Appendix 2, available at www.cmaj.ca/ lookup/suppl/doi:10.1503/cmaj.141525/-/DC1).
Competing interests: None declared.

This article has been peer reviewed.

The authors have obtained patient consent.

Correspondence to: Yi-Da Li, blueway810@yahoo.com.tw CMAJ 2015. DOI:10.1503 /cmaj.141525 


\section{Discussion}

Platypnea-orthodeoxia syndrome is an uncommon clinical syndrome that usually presents with a cardinal symptom (platypnea: dyspnea in an upright posture relieved by recumbency) and sign (orthodeoxia: hypoxemia in an upright position).

The exact cause of platypnea-orthodeoxia syndrome is not fully understood, possibly because patients do not present with pulmonary hypertension and right chamber pressure is normal. ${ }^{1,2}$ Both anatomic and functional abnormalities must be present to cause platypnea-orthodeoxia syndrome. ${ }^{3}$ The anatomic component refers to the presence of any structural defect which allows interatrial communication, including patent foramen ovale, atrial septal defect and fenestrated atrial septal aneurysm. The functional component refers to any condition that may cause an atrial septum deformity, leading to intracardiac shunting while the patient is in the upright position. ${ }^{3}$ We speculate that the development of the substantial intracardiac shunt in our patient's case was likely the result of dilatation of the ascending aorta and kyphoscoliosis, which has been reported to cause abnormal orientation of the heart (Figure 2). ${ }^{4}$

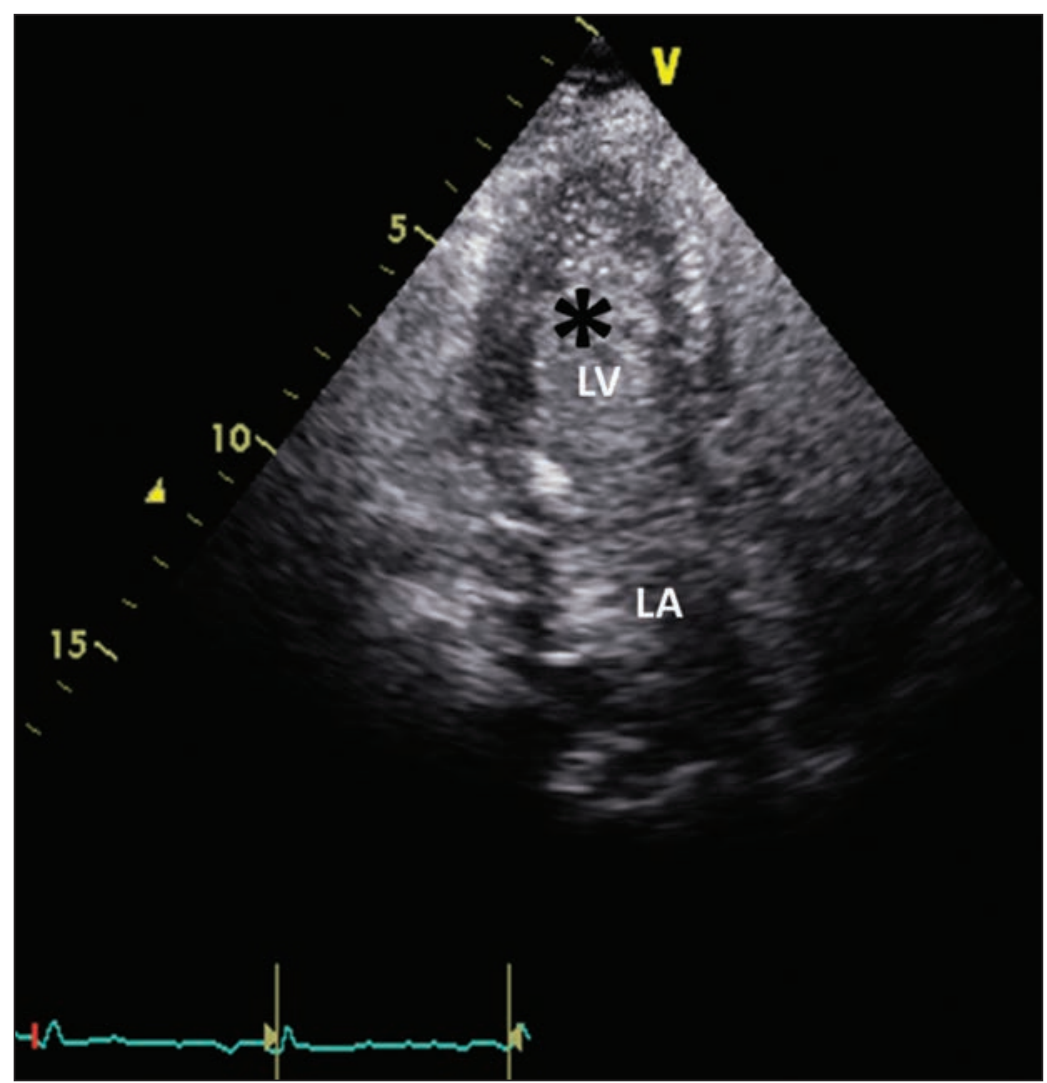

Figure 1: Bubble echocardiogram from a 73-year-old woman with progressive shortness of breath. Bubbles (asterisk) were seen in the left atrium (LA) and left ventricle (LV) within three cardiac cycles. We diagnosed platypneaorthodeoxia syndrome.

\section{Patent foramen ovale}

Although patent foramen ovale may be clinically silent for decades, patients with this defect may present with cardiac platypnea-orthodeoxia syndrome. It has been proposed that the intracardiac communication caused by a patent foramen ovale results in transiently elevated right chamber pressure, and the "flow" phenomenon (i.e., distortion of anatomy favouring passage of blood to the interatrial communication) may lead to the development of platypnea-orthodeoxia syndrome. ${ }^{2,45}$ In addition, aortic changes (e.g., elongation and dilatation) may cause the atrial septum to become horizontal, thereby promoting a more direct pathway for blood flow from the vena cava to the interatrial communication, despite the absence of a pressure gradient. ${ }^{4,5}$ Moreover, it has been proposed that an upright position could stretch the interatrial communication, allowing more streaming of venous blood from the inferior vena cava through the defect. ${ }^{3}$

\section{Investigations}

A noninvasive workup including serial oxygen saturation measurements with standard pulse oximetry and echocardiography are usually sufficient to establish the diagnosis of platypneaorthodeoxia syndrome. Contrast transthoracic echocardiography helps to detect the presence of a right-to-left shunt, whereas transesophageal echocardiography is better for assessing atrial anatomy. ${ }^{6}$ The gold standard diagnostic test for platypnea-orthodeoxia syndrome remains cardiac catheterization with oxygen measurement directly in the left atrium. ${ }^{6,7}$

\section{Management}

The definitive treatment for cardiac platypneaorthodeoxia syndrome is percutaneous closure of the interatrial communication, which appears to be safe and efficient, with a low rate of complication. ${ }^{6,8}$ Other possible diagnoses, such as pulmonary hypertension, should be excluded before closure of the defect to avoid clinical deterioration of the patient's condition after correction of right-to-left-shunt.

In our patient's case, we were concerned about the effects of a three-hour journey and reviewed the literature for possible pharmacological interventions to use as a bridge to surgery. However, we found little information. Only one case reported the use of a potent $\alpha$-adrenergic agonist (metaraminol) to reverse the intracardiac shunt by causing a sharp rise of left ventricular end-diastolic pressure and left atrial pressure in occult atrial septal defect. ${ }^{9}$

We attempted to increase left ventricular end diastolic pressure using vasopressors, including 
dopamine and norepinephrine, to temporarily reverse the intracardiac shunting. Norepinephrine is a strong $\alpha$-receptor agonist and a weak $\beta_{1}$-adrenergic agonist, thus producing a dosedependent systemic vascular resistance and modest increase in cardiac output (Figure 3).

In contrast, dopamine is a dose-dependent vasopressor that has various hemodynamic effects. At low doses $(<3 \mu \mathrm{g} / \mathrm{kg}$ per min), dopamine increases blood flow in renal and splanchnic regions. ${ }^{10}$ At moderate doses $(3-10 \mu \mathrm{g} / \mathrm{kg}$ per min), it stimulates $\beta$ receptors, increasing myocardial contractility and heart rate while causing peripheral vasodilation. ${ }^{10}$ At higher doses ( $>10 \mu \mathrm{g} / \mathrm{kg}$ per min), dopamine activates $\alpha$ receptors, leading to systemic and pulmonary vasoconstriction. ${ }^{10}$ However, varying serum drug concentrations can result when dopamine is administered by weight in various patients. ${ }^{11}$

Norepinephrine successfully reversed the intracardiac shunting in our patient; this effect was probably due to a more selective action of norepinephrine on peripheral vasoconstriction (Figure 3). The moderate dose of dopamine we used likely caused sinus tachycardia or tachyarrhythmia in our patient, which lead to her chest discomfort.

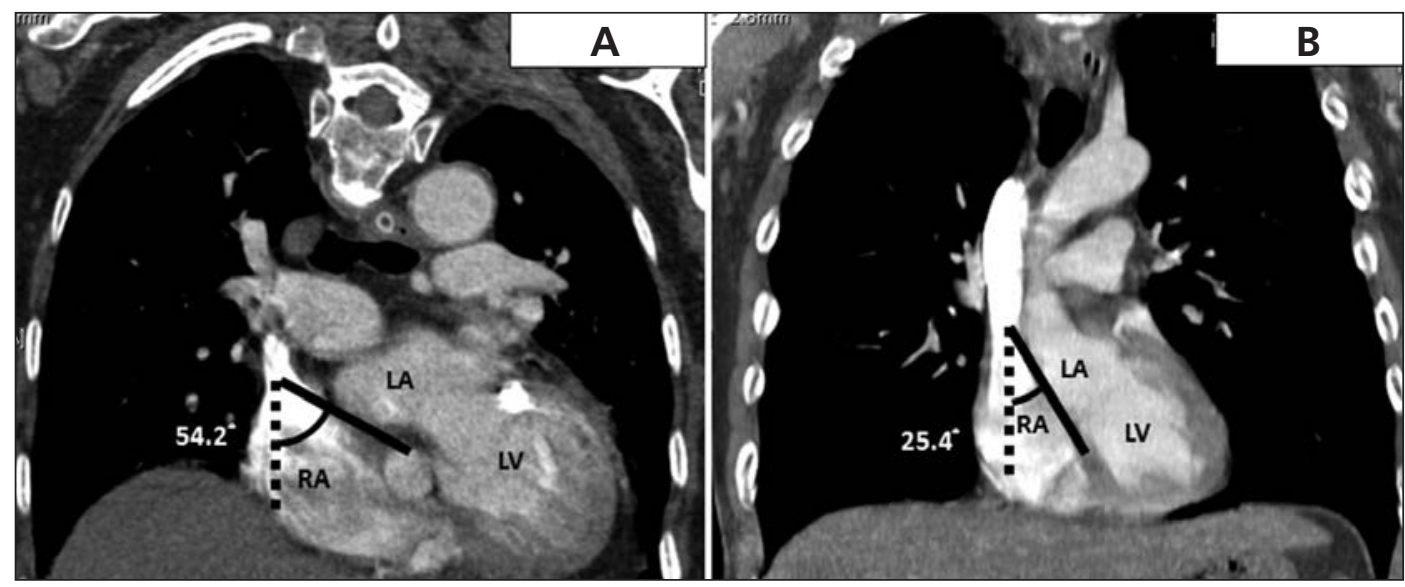

Figure 2: Computed tomography showing the angle between the interatrial septum (solid line) and the sagittal axis (dotted line). The angle in our patient's case measured $54.2^{\circ}(\mathrm{A})$, whereas $25.4^{\circ}$ is considered normal (B). The horizontal orientation of the atrial septum promotes a more direct pathway for venous blood flow, which may directly hit the interatrial septum and cause intracardiac shunting, even without a pressure gradient. LA = left atrium, LV = left ventricle, RA = right atrium.

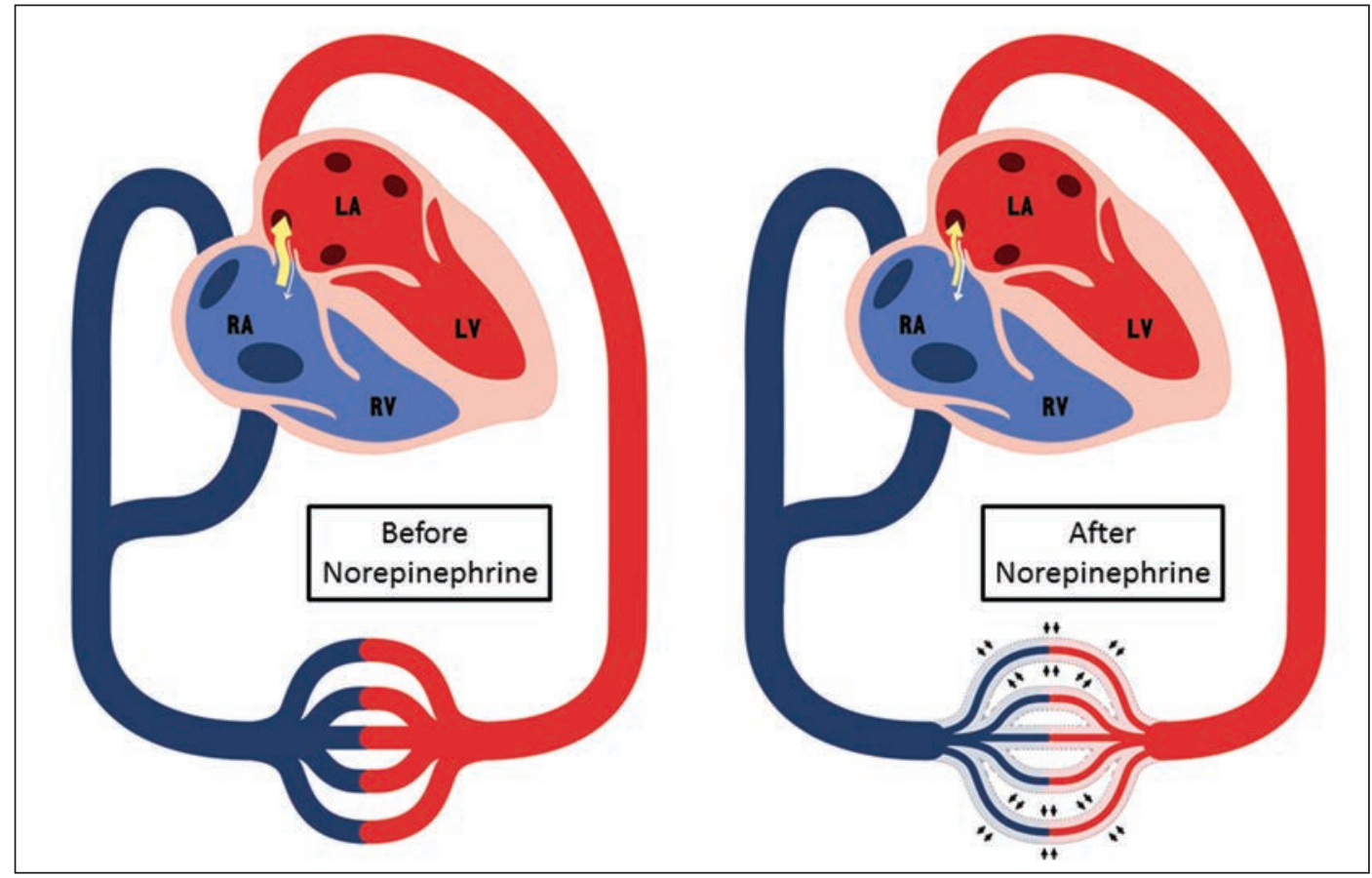

Figure 3: Hemodynamic changes before and after the use of norepinephrine in a patient with platypneaorthodeoxia syndrome. $\mathrm{LA}=$ left atrium, LV = left ventricle, $\mathrm{RA}=$ right atrium, $\mathrm{RV}=$ right ventricle. 


\section{References}

1. Pierce CW. Platypnea-orthodeoxia syndrome in an elderly woman with a patent foramen ovale. Can J Cardiol 2010;26:213-4

2. Rodrigues P, Palma P, Sousa-Pereira L. Platypnea-orthodeoxia syndrome in review: defining a new disease? Cardiology 2012; 123:15-23.

3. Cheng TO. Mechanisms of platypnea-orthodeoxia: What causes water to flow uphill? Circulation 2002;105:e47

4. Kusunose K, Yamada H, Todoroki T, et al. Platypnea-orthodeoxia syndrome associated with patent foramen ovale and aortic ectasia. Echocardiography 2009;26:114-7.

5. Bertaux G, Eicher JC, Petit A, et al. Anotomic interaction between the aortic root and the atrial septum: a prospective echocardiographic study. J Am Soc Echocardiogr 2007;20:409-14.

6. Blanche C, Noble S, Roffi M, et al. Platypnea-orthodeoxia syndrome in the elderly treated by percutaneous patent foramen ovale closure: a case series and literature review. Eur J Intern Med 2013;24:813-7.

7. Desouza KA, Saraswat S, DeSouza SA, et al. Platypnea-orthodeoxia syndrome: a diagnostic challenge. South Med J 2009;102:1046-8.

8. Guérin P, Lambert V, Godart F, et al. Transcatheter closure of patent foramen ovale in patients with platypnea-orthodeoxia: results of a multicentric French registry. Cardiovasc Intervent Radiol 2005;28:164-8.

9. Mackenzie IM, Banning A, Dyar O. Pharmacologic exposure of an occult atrial septal defect. Crit Care Med 2001;29:1832-4.

10. Kellum JA. J MD. Use of dopamine in acute renal failure: a meta-analysis. Crit Care Med 2001;29:1526-31.

11. MacGregor DA, Smith TE, Prielipp RC, et al. Pharmacokinetics of dopamine in healthy male subjects. Anesthesiology 2000;92:338-46.

Affiliations: School of Medicine (Ng, Li), Tzu Chi University, Hualien, Taiwan; Departments of Internal Medicine (Ng, Li) and Cardiology (Li), Dalin Tzu Chi Hospital, Buddhist Tzu Chi Medical Foundation, Chiayi, Taiwan
Contributors: Both of the authors contributed to the conception, writing and revising of the article. Both of the authors approved the final version to be published and agree to act as guarantors of the work.

The section Cases presents brief case reports that convey clear, practical lessons. Preference is given to common presentations of important rare conditions, and important unusual presentations of common problems. Articles start with a case presentation (500 words maximum), and a discussion of the underlying condition follows (1000 words maximum). Visual elements (e.g., tables of the differential diagnosis, clinical features or diagnostic approach) are encouraged. Consent from patients for publication of their story is a necessity. See information for authors at www.cmaj.ca.

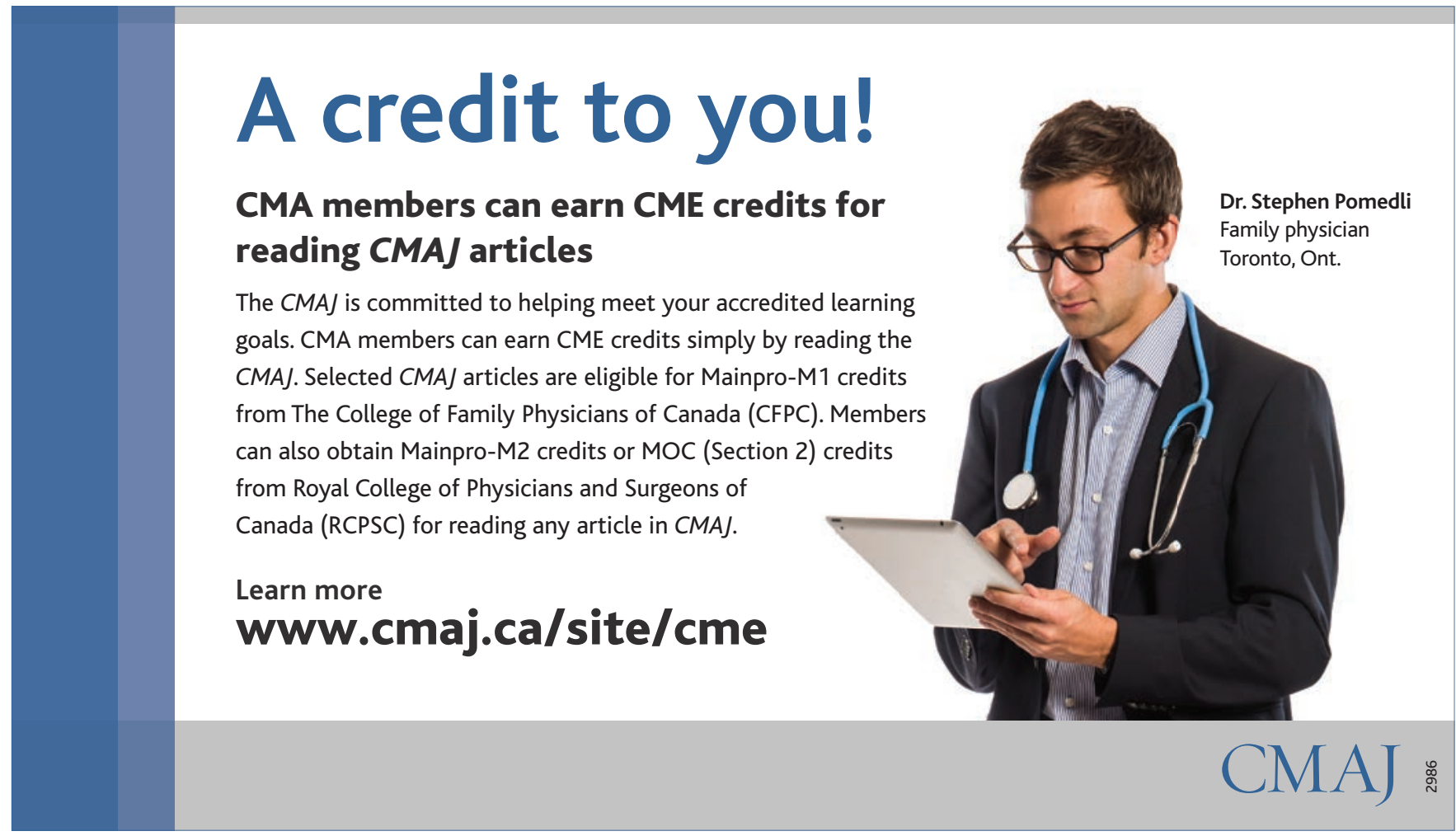

